\title{
Cefaleias na gravidez: um caso clínico
}

Ana Raquel Marques, * Ana Maria Falcão**

\section{RESUMO}

Enquadramento: A cefaleia é um sintoma comum nos cuidados de saúde primários (CSP). Pode ter características sugestivas de uma cefaleia secundária grave, devendo o médico de família (MF) estar atento aos sinais de alarme. Sendo assim, perante um sintoma inespecífico, as competências nucleares do MF capacitam-no para gerir a incerteza, facilitando a gestão das várias possibilidades.

Descrição de caso: Sexo feminino, 21 anos, antecedentes de disseção aórtica aos nove anos, sem MF na Unidade Funcional. A 01/09 recorre a consulta de intersusbstituição (CIS), grávida de 12 semanas, por cefaleias com 2,5 semanas, holocranianas e quase diárias. Foi-lhe indicado paracetamol em SOS. Por antecedentes cardiovasculares importantes foi referenciada a consulta hospitalar de obstetrícia. A 14/09 recorre a CIS por manter cefaleias holocranianas de predomínio frontal, de caráter pulsátil, com fonofobia, fotofobia e osmofobia, sem cedência ao analgésico. É encaminhada para o serviço de urgência (SU), onde foi observada por neurologia que considerou tratar-se de uma enxaqueca sem aura. A 01/10 teve consulta de obstetrícia, referindo novamente cefaleias intensas com reforço da toma de analgésico. A 07/10, agora com 17 semanas de gestação, recorre a CIS por manter cefaleias frontais. Por quadro arrastado, com recurso a múltiplas consultas com médicos diferentes, opta-se por contactar telefonicamente o neurologista de urgência. No SU, a angio-ressonância magnética (RM) revelou trombose venosa cerebral do seio lateral esquerdo. Foi internada na Unidade de Cuidados Intermédios Polivalentes e iniciou terapêutica com enoxaparina 12/12h. Discussão: A prevalência das cefaleias nos CSP é alta e, na maioria dos casos, sem sinais de alarme. A continuidade de cuidados, abordagem abrangente, modelação holística e a capacidade de gestão de cuidados - características da medicina geral e familiar -, são essenciais na avaliação destes utentes e conferem ao MF clara vantagem sobre os restantes especialistas na resolução de problemas complexos ou indiferenciados. Este caso vem demonstrar, uma vez mais, a importância de todos terem um MF.

Palavras-chave: Cefaleias; Gravidez; Trombose venosa cerebral.

\section{DESCRIÇÃO DO CASO}

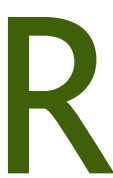

elata-se o caso de uma utente do sexo feminino, de 21 anos, empregada hoteleira, com o $12^{\circ}$ ano de escolaridade, solteira, integrada em família nuclear na fase I do ciclo de vida de Duvall. Trata-se de uma utente que vive com o companheiro, em habitação com boas condições, próxima da residência dos pais de ambos. Tanto a utente como o companheiro têm atividade laboral remunerada e uma escolaridade de 12 anos. Como empregada hoteleira tinha um horário bastante rígido, por vezes com necessidade de carregar materiais pesados. Tinha antece-

*Médica Assistente de Medicina Geral e Familiar. USF Lavra, Unidade Local de Saúde de Matosinhos

**Médica Interna de Medicina Geral e Familiar. USF Calâmbriga. Agrupamento de Centros de Saúde de Entre Douro e Vouga II - Aveiro Norte dentes de cirurgia corretiva a disseção aórtica aos nove anos. Esta utente não tinha médico de família atribuído na Unidade Funcional (UF).

Recorreu a consulta de intersubstituição (CIS) na UF a 28/07/2015 por tonturas, náuseas e cefaleias holocranianas com uma semana de evolução e com suspeita de gravidez por amenorreia. O exame objetivo, incluindo exame neurológico sumário, foi descrito como sem alterações. Realizou teste de gravidez na UF, que foi positivo, tendo iniciado a toma de ácido fólico e iodo na dose recomendada pelo Programa Nacional para a Vigilância da Gravidez de Baixo Risco da DireçãoGeral da Saúde. ${ }^{1}$ Foi explicado à utente que deveria recorrer à CIS, com diferentes médicos de família, para nova avaliação e para saber o resultado do estudo analítico, do rastreio pré-natal e do estudo ecográfico de $1^{\circ}$ trimestre que iria realizar no hospital da área de resi- 
dência, como protocolado entre o ACeS e o serviço de obstetrícia, que disponibiliza a todas as grávidas no $1^{\circ}$ trimestre que recorram às suas unidades de atendimento o rastreio pré-natal das trissomias 13, 18 e 21, através do estudo bioquímico e ecográfico. Quanto às cefaleias foi-lhe indicado tomar paracetamol em SOS na dose de $1000 \mathrm{mg}$, três vezes ao dia.

A 01/09, com gestação de 12 semanas, recorreu a CIS por cefaleia com cerca de duas semanas e meia de evolução, holocraniana, quase diária e sem melhoria com analgésico prescrito. Nesta consulta foram averiguados os antecedentes pessoais da utente de cirurgia corretiva a disseção aórtica aos nove anos. Por antecedentes cardiovasculares importantes, o médico que observou a utente optou por referenciar a mesma para a consulta hospitalar de obstetrícia. A 14/09, com 14 semanas de gestação, recorreu novamente a CIS por manter cefaleia intensa holocraniana, mas de predomínio frontal, constante, de caráter pulsátil, com fonofobia, fotofobia e osmofobia, com interferência na sua atividade laboral e sem cedência à toma de paracetamol e sem fatores de agravamento. Não impedia o sono. Negava antecedentes pessoais ou familiares de enxaqueca. Foi encaminhada para o serviço de urgência (SU) do hospital da área de residência. No SU foi observada por obstetrícia que pediu colaboração a neurologia. À observação não apresentava alterações visuais, fundoscopia normal, sem alterações dos nervos cranianos, da força e tónus muscular, reflexos osteotendinosos presentes e simétricos, sem alterações da sensibilidade álgica à picada, sem alterações da sensibilidade postural, sem alterações cerebelosas ou sinais de irritação meníngea. Realizou, ainda no SU, a toma de ácido acetilsalícilico oral com melhoria da sintomatologia. Assim, o neurologista considerou que a cefaleia cumpria características de enxaqueca sem aura e que não existiam, nem na história clínica nem no exame neurológico, sinais de alarme para cefaleia secundária. Foi considerado ainda que a duração prolongada da cefaleia se devia ao tratamento com paracetamol, por norma pouco eficaz na enxaqueca, tendo tido alta para o domicílio com indicação para a toma esporádica de anti-inflamatório não esteróide, no caso de dor intensa, até às 32 semanas de gestação.

A 01/10, com gestação de 16 semanas e três dias, teve consulta de obstetrícia, referindo novamente cefaleias muito intensas, tendo sido reforçada a toma de paracetamol ou de ibuprofeno até $1200 \mathrm{mg}$ por dia, como orientado pela neurologia.

Por manutenção das cefaleias já com dois meses de evolução, recorreu novamente a CIS na UF a 07/10, com gestação de 17 semanas, associada a ocasionais alterações visuais (visão nublada), tendo sido observada pela médica interna de apoio à CIS. Referia não cumprir o ibuprofeno por receio da toma durante a gravidez, mas tomava paracetamol de $8 / 8 \mathrm{~h}$ sem alívio da sintomatologia. Ao exame objetivo, normotensa, não revelou edemas dos membros inferiores e apresentou tira-teste de urina sem alterações. Por quadro arrastado, com recurso a múltiplas consultas com médicos diferentes, opta-se por contactar telefonicamente o neurologista de urgência para expor o caso, concordando o colega que a utente apresentava sinais de alarme e que deveria ser encaminhada para o SU para realizar uma ressonância magnética (RM) cerebral. Ao exame neurológico no SU apresentava ligeiro edema da papila traduzido por discreto apagamento dos vasos, bilateralmente. Na angio-ressonância magnética (angio-RM), para tempos venosos, observaram-se "acentuadas irregularidades do seio lateral e do seio sigmóide esquerdos, com maior acentuação das veias corticais, a sugerir áreas de trombose do seio e, eventualmente, com alguma recanalização". Em conclusão, tratava-se dum quadro de trombose venosa cerebral do seio lateral esquerdo, aparentemente estável, sem lesões parenquimatosas por seio parcialmente recanalizado/trombosado. A utente foi internada na Unidade de Cuidados Intensivos Polivalentes (UCIP) e iniciou terapêutica com enoxaparina $12 / 12 \mathrm{~h}$, que atualmente mantém. O restante estudo analítico (hemograma, função renal, função hepática, ionograma e sumário de urina) sem alterações mas mantém estudo e acompanhamento hospitalar por etiologia trombofílica potencial a esclarecer. A utente manteve seguimento da sua gravidez a nível hospitalar, mas voltou à UF a agradecer a disponibilidade da médica interna tornando-se utente frequentadora da lista da sua orientadora de formação, com manutenção do seguimento da gravidez também na Unidade. A utente teve um parto eutócico, de um menino, às 39 semanas, que decorreu sem qualquer intercorrência e o período pós-natal está a correr de forma harmoniosa, não tendo sido registadas mais queixas até à data. 


\section{DISCUSSÃO}

Desde o início do quadro, a utente recorreu a múltiplas consultas num espaço de dois meses (tempo de evolução da cefaleia): quatro consultas de intersubstituição com vários médicos, uma consulta hospitalar de obstetrícia e duas idas ao SU. O programa nacional de vigilância de gravidez de baixo risco ${ }^{1}$ define a periodicidade das consultas a realizar, a metodologia a adotar em cada consulta (anamnese, exame físico, exames auxiliares de diagnóstico, terapêutica, educação para a saúde) e os critérios de referenciação para os cuidados de saúde secundários. O papel do MF no seguimento destas gravidezes é, assim, contribuir para um futuro mais saudável da população, atuando desde o início do ciclo de vida através de cuidados abrangentes, antecipatórios e individualizados, numa perspetiva de participação ativa das mulheres / famílias. A obtenção de uma história clínica detalhada, em particular da história obstétrica pregressa e a observação clínica, são fundamentais na definição dos cuidados a prestar e requerem disponibilidade e uma continuidade de cuidados e de vigilância. Além disso, todas as informações recolhidas sobre saúde, estilos de vida, comportamentos e ambiente psicossocial são elementos essenciais para a prossecução deste objetivo.

Apesar de ser uma gravidez não planeada, tanto o casal como a família mais próxima vivia a gravidez de forma intensa e encaravam o novo bebé como parte integrante do agregado familiar. Era motivo de alegria e de união familiar. A doença inesperada da utente e a possível interferência com a gravidez causaram grande ansiedade nos elementos da família. No caso desta utente, a inexistência de um MF atribuído dificultou toda a vigilância da gravidez que, desde o início, foi assumida como uma gravidez de baixo risco pela escala modificada de Goodwin, sem agendamento de consultas de vigilância, sem centralização da informação e sem tempo de consulta adequada para estabelecer uma relação de confiança entre o profissional de saúde e a grávida. Esta relação facilitaria a expressão de ideias, expectativas, fantasias, sentimentos (positivos e negativos) e competências inerentes à gravidez, nascimento e parentalidade. O recurso frequente aos cuidados de saúde com acesso a médicos diferentes contribuiu para o aumento da ansiedade no seio familiar e teve também impacto na atividade profissional da grávida, que se ausentava com frequência do seu local de trabalho. No entanto, a entidade patronal desde o início de todo o processo que se revelou compreensiva e facilitadora do acesso aos cuidados de saúde. Para além de uma gravidez não vigiada de forma adequada, a utente apresentava ainda queixas de cefaleia com dois meses de evolução, coincidentes com o diagnóstico da gravidez.

No que à acessibilidade aos serviços de saúde diz respeito, apesar de não existir agendamento de consultas, a utente teve consulta sempre que necessário e justificado. No entanto, o processo de diagnóstico tornou-se moroso. Se por um lado, as queixas eram inespecíficas, por outro, o facto de ter sido um seguimento realizado por vários profissionais poderá ter quebrado uma eventual linha condutora que pudesse existir.

O processo terapêutico apresentou-se também como um entrave. Algumas das propostas de tratamento foram recusadas pela utente pelo facto de estar grávida e de ter receio de reações adversas no bebé. Efetivamente, o ibuprofeno está contraindicado a partir do terceiro trimestre de gravidez, ${ }^{2}$ o que restringia a escolha da terapêutica. Por isso, não alcançando o efeito terapêutico acabava por desistir da medicação instituída.

Nos CSP são vulgares quatro tipos de cefaleia (enxaqueca, cefaleia tipo tensão, cefaleia em salvas e cefaleia por uso excessivo de medicamentos). Todos têm uma base neurobiológica, são incapacitantes e diminuem a qualidade de vida. ${ }^{3} \mathrm{~A}$ anamnese assume, desta forma, grande importância no diagnóstico das cefaleias e na distinção entre causas primárias e secundárias, por exemplo, ao uso excessivo de medicamentos ou a outras situações de maior gravidade, como eventos tromboembólicos ou lesões ocupantes de espaço. Não há exames complementares de diagnóstico patognomónicos. A história clínica deverá detetar quaisquer características sugestivas de uma cefaleia secundária grave. $^{3}$

Constituem sintomas e sinais de alarme na história e/ou no exame: ${ }^{3}$ cefaleia de novo ou inesperada num determinado doente ou com novas características; cefaleia explosiva primária (cefaleia intensa, de início súbito ou «explosivo»), que poderá indicar hemorragia subaracnóidea; cefaleia com aura atípica (duração $>1$ hora ou que inclui parésia), podendo ser um sintoma de acidente isquémico transitório (AIT) ou acidente vascular 
cerebral (AVC); aura sem cefaleia, sem história prévia de enxaqueca com aura, podendo ser um sintoma de AIT ou AVC; aura que ocorre pela primeira vez numa doente, enquanto esta toma contracetivos orais combinados, indicando risco de AVC; cefaleia de novo num paciente com idade superior a 50 anos, podendo ser um sintoma de arterite temporal ou tumor intracraniano, ou numa criança antes da puberdade; cefaleia progressiva que piora ao longo de semanas ou mais e que não cede a terapêutica adequada; cefaleia associada a, ou agravada por, mudanças de posição ou outros movimentos que aumentam a pressão intracraniana, podendo indicar a presença de um tumor intracraniano; cefaleia de novo num doente com história de cancro, infeção ou imunodeficiência por VIH; febre inexplicável associada a cefaleia, podendo indicar meningite e ainda sinais neurológicos focais associados a cefaleia.

Numa fase inicial, a utente não apresentava cefaleia com sinais de alarme mas, com a progressão do quadro, surgiram alguns sintomas e sinais que alteraram a gravidade do mesmo. Surgiram alterações visuais (visão nublada) e um ligeiro edema da papila traduzido por discreto apagamento dos vasos, bilateralmente, sem défices focais associados (apenas possível valorizar através da observação com oftalmoscópio e conhecimento adequado da sua utilização).

Relativamente ao tratamento da cefaleia, todos os indivíduos devem progredir numa escalada de tratamentos abortivos ou sintomáticos (abordagem por patamares), tratando, geralmente, três crises por patamar, antes de passar ao próximo. Se aplicada corretamente, esta é uma boa estratégia para proporcionar os cuidados personalizados mais eficazes e económicos. ${ }^{3} \mathrm{O}$ primeiro patamar é constituído por terapêutica sintomática, não específica: analgésico simples ou anti-inflamatório, complementado, se necessário, por um antiemético. Neste contexto, inicialmente foi-lhe receitado paracetamol, ao qual a cefaleia não cedia. Posteriormente, a terapêutica foi otimizada com ibuprofeno, mas não cumprida pela grávida por receio das consequências para a gravidez. O segundo patamar consiste em terapêutica específica e preventiva, nomeadamente triptanos. ${ }^{3}$

Entre as razões que levam à referenciação para os cuidados de saúde secundários encontram-se: as dúvidas de diagnóstico após a anamnese e exame físico com- pleto, a suspeita de uma cefaleia de causa secundária e os casos que exijam uma investigação para excluir patologias graves (poderá ser necessária a referenciação imediata). ${ }^{3}$ Foi o caso desta grávida que, por dúvidas no diagnóstico, com suspeita de causa secundária grave foi referenciada ao SU ao cuidado de neurologia.

Várias evidências sugerem relação entre cefaleia e o ciclo de vida normal das mulheres, incluindo importantes fases de alteração hormonal: menarca, gravidez, uso de anticontracetivos orais, menopausa e terapia de substituição hormonal. ${ }^{4}$ No que diz respeito à cefaleia na gravidez, se a mulher já sofria de cefaleias anteriormente, as suas características podem alterar durante a gestação. ${ }^{4}$ Apenas $7 \%$ das mulheres descrevem surgimento de cefaleia pela primeira vez na gravidez. ${ }^{2}$ Há, no entanto, relato de enxaqueca iniciando-se pela primeira vez na gravidez, principalmente enxaqueca com aura. ${ }^{4}$ Esta situação poderia corresponder ao caso da utente em questão, pois nos antecedentes não há relato de história de cefaleias, como descrito anteriormente. Contudo, é-nos descrita uma enxaqueca sem aura, que não é a mais comum na gravidez. Outro aspeto a considerar é que, se a cefaleia não melhorar até final do $1^{\circ}$ trimestre, é pouco provável que melhore no tempo restante da gravidez. ${ }^{2}$ No caso descrito, as queixas mantiveram-se mesmo após o $1^{\circ}$ trimestre e sem sinais de melhoria.

Quanto ao tratamento das cefaleias durante a gravidez, há alguns medicamentos que podem ser utilizados de forma segura. No entanto, esta questão deve ser sempre ponderada de forma individual, tendo em conta características da grávida e da cefaleia. ${ }^{2}$

Para a dor leve ou moderada e de início lento, o paracetamol, anti-inflamatórios não esteroides e opióides poderão ser tomados, com moderação, no início da gravidez. ${ }^{2} \mathrm{O}$ uso de anti-inflamatórios não esteroides está contraindicado no $3^{\circ}$ trimestre devido à constrição ou eventual encerramento do canal arterial fetal ou enterocolite necrosante. Embora o paracetamol possa ser menos eficaz do que anti-inflamatórios não esteroides ou triptanos, no tratamento da enxaqueca é considerado um medicamento «seguro» na gravidez (categoria de risco B). ${ }^{2}$ Ainda assim, os doentes (neste caso, a grávida) devem ser advertidos a respeito da toxicidade hepática com o uso excessivo. Os opióides têm uma eficácia limitada no tratamento da cefaleia, embora os de 
curta ação como a codeína possam ser úteis no tratamento agudo, em associação com o paracetamol. ${ }^{2}$

Para a dor moderada a grave, ou que aumenta rapidamente de intensidade, ou numa história de enxaqueca com significativa interferência nas atividades de vida diária, podem ser considerados os triptanos. Embora a gravidez constitua uma contraindicação para o seu uso, não existe evidência definitiva de teratogenicidade. Em grávidas com enxaqueca grave, o benefício poderá ser superior aos riscos. ${ }^{2}$ No entanto, mais estudos são necessários com o intuito de encorajar ou desencorajar o seu uso.

A trombose venosa cerebral (TVC) é uma situação relativamente rara, com uma incidência anual estimada de três a quatro casos por milhão de habitantes na população em geral, ${ }^{5}$ sendo que $75 \%$ dos doentes adultos são mulheres. ${ }^{6} \mathrm{~A}$ incidência de TVC na gravidez é variável com o nível socioeconómico do país. Nos países desenvolvidos, a incidência é de 10 a 20 casos por 100.000 mulheres grávidas, sendo responsável por $6 \%$ das mortes maternas ${ }^{7}$ e correspondendo a 5 a $10 \%$ de todas as TVC. ${ }^{8}$

Os fatores causais podem ser divididos em locais ou sistémicos, ${ }^{7}$ de que são exemplos estados pró-trombóticos genéticos (défice antitrombina III, proteína C e S) ou adquiridos (gravidez e puerpério), infeções (otite, mastoidite), doenças inflamatórias (lúpus eritematoso sistémico, sarcoidose), doenças hematológicas, medicamentos, entre outros. ${ }^{6}$ No caso desta utente, a causa da TVC poderá ter sido o fator adquirido gravidez; no entanto, estudos decorrem no sentido de averiguar se se trata de uma trombofilia.

O motivo da incidência de TVC na gravidez relaciona-se com o facto de esta ser um estado de hipercoagulabilidade, observando-se alterações no sistema de coagulação, sistema fibrinolítico, fluxo venoso e paredes dos vasos, que pode predispor à trombose. ${ }^{7}$ As manifestações clínicas dependem da extensão e localização do processo trombótico, bem como da existência de circulação venosa colateral adequada. ${ }^{7}$ As cefaleias são o sintoma mais frequente $(75 \text { a } 95 \% \text { dos casos })^{6} \mathrm{e}$ mais precoce de TVC. O facto de não terem características específicas e de poderem não estar associadas a outros sinais neurológicos tornam o diagnóstico da doença mais difícil. ${ }^{6-7}$ Assim, o diagnóstico correto é, por vezes, feito com demora considerável, necessitan- do, além disso, de exames de neuro imagem para a sua confirmação. ${ }^{9}$ Como foi constatado no caso descrito, a doente foi observada várias vezes em consultas de vários profissionais, até que fosse estabelecido o diagnóstico de TVC. A cefaleia não era característica e não apresentava sintomas ou sinais neurológicos associados, numa fase inicial, provavelmente pela existência de circulação venosa colateral e pela recanalização parcial do seio.

A RM cerebral, especialmente a angio-RM, e a angiografia cerebral são os exames auxiliares que conduzem ao diagnóstico de TVC. ${ }^{9}$ São aqui de destacar as acentuadas irregularidades do seio lateral e do seio sigmoide esquerdos, com maior acentuação das veias corticais, a sugerir áreas de trombose do seio e eventualmente com alguma recanalização descritas na angioRM da utente e que levaram ao diagnóstico de TVC.

O tratamento da TVC inclui anticoagulação plena na fase aguda, tratamento de crises epiléticas e controlo de hipertensão intracraniana, se existirem. ${ }^{10} \mathrm{~A}$ finalidade da anticoagulação é evitar a progressão do trombo, ajudando na recanalização venosa. ${ }^{10}$

Este caso clínico pretende, assim, alertar para alguns fatores que podem ter dificultado o encaminhamento mais precoce e adequado desta utente:

- A cefaleia trata-se de um sintoma inespecífico e muito frequente nos CSP, que pode ter sinais de alarme que devem ser ativamente pesquisados e que podem não ocorrer facilmente ao MF;

- Apesar de estar descrito que a enxaqueca se pode iniciar pela primeira vez na gravidez, principalmente enxaqueca com aura, o acompanhamento e vigilância da gravidez permitem ao MF avaliar a evolução e o aparecimento de sinais ou sintomas de alarme;

- A realização de um exame objetivo neurológico sistemático em todas as consultas perante uma queixa persistente de cefaleia é essencial para a procura de sintomas neurológicos que podem ser indicativos de causa grave de cefaleia;

- A importância do MF ter conhecimentos atualizados sobre as causas de cefaleia, diagnóstico, tratamento e diagnósticos diferenciais.

O relato deste caso visa ainda salientar a importância de todos os utentes serem devidamente assistidos por um MF que, pelas suas competências nucleares, é 
capaz de utilizar eficientemente os recursos de saúde, coordenando a prestação de cuidados e gerindo a interface com outras especialidades, assumindo um papel de advogado do paciente, sempre que necessário, perante a gestão da doença que se apresenta de forma indiferenciada, numa fase precoce da sua história natural e que pode necessitar de intervenção urgente. A sua visão holística do doente permite desenvolver uma abordagem centrada na pessoa e ter um processo de consulta singular em que se estabelece uma relação ao longo do tempo, através de uma comunicação médicopaciente efetiva, e de ser responsável pela prestação de cuidados continuados longitudinalmente. Citando o Dr. Margarte Chan, diretor geral da Organização Mundial da Saúde, primary care is our best hope for the future. Family doctors are our rising stars for the future.

O MF, lidando com uma alta prevalência de queixas, muitas vezes indiferenciadas, como a cefaleia, deve valorizar a sintomatologia e procurar ativamente sinais de alarme, com vista ao possível diagnóstico e tratamento de causas secundárias graves, com risco de incapacidade e mesmo com perigo de vida, que necessitam de referenciação urgente.

No caso descrito, a interna foi a médica que observou a utente na última consulta na Unidade e que, por persistência da cefaleia, contactou o colega da especialidade para encaminhamento da utente. A persistência das queixas ao longo do tempo (um dos sinais de alarme descrito na literatura $)^{3}$ foi, de facto, o sinal chave gerido por esta médica.

A reflexão sobre este caso clínico desencadeou uma importante mudança na gestão/cuidados aos doentes sem médico no caso particular desta unidade de saúde. Foi criada uma consulta específica para os utentes sem médico de família com necessidades específicas de vigilância (saúde materna, planeamento familiar e crianças até aos dois anos). A situação clínica da grávida e a fragilidade que acarretava à mesma e ao seu agregado familiar motivaram a inclusão da utente da lista da orientadora de formação da interna. A utente manteve seguimento na Unidade com a nova médica de família que se manteve sempre atualizada sobre o de- senvolvimento da gravidez e de todo o seguimento no hospital. Às 39 semanas nasceu um menino saudável, de parto eutócico e não houve, até à data, registo de intercorrências.

\section{REFERÊNCIAS BIBLIOGRÁFICAS}

1. Direção-Geral da Saúde. Programa nacional para a vigilância de gravidez de baixo risco. Lisboa: DGS; 2016.

2. Lucas $\mathrm{S}$. Medication use in the treatment of migraine during pregnancy and lactation. Curr Pain Headache Rep. 2009;13(5):392-8.

3. European Headache Federation. Princípios europeus da abordagem das cefaleias comuns nos cuidados de saúde primários [Internet]. Lisboa: Sociedade Portuguesa de Cefaleias; 2010. Available from: http://ehforg.org/wp-content/uploads/2013/12/European-Principles-of-Management-of-Common-Headache-Disorders-in-Primary-Care_Portuguese-Translation.pdf

4. Camargo $\mathrm{CH}$. Cefaleias e gestação: um estudo clínico epidemiológico em 100 mulheres em um hospital universitário [Dissertation]. Florianópolis: Universidade Federal de Santa Catarina; 1997.

5. Matos LC, Martins B, Canto-Moreira N, Gomes A, Martins I, Capelo J, et al. Trombose de seios durais [Dural sinus thrombosis]. Acta Med Port. 2007;20(4):369-73. Portuguese

6. Stam J. Thrombosis of the cerebral veins and sinuses. N Engl J Med. 2005;352(17):1791-8.

7. Ferreira MM, Rios AC, Fragata I, Baptista JT, Manaças R, Reis J. Aspectos imagiológicos da trombose venosa cerebral numa mulher grávida [Cerebral venous thrombosis imagiologic features in a pregnant woman]. Acta Med Port. 2011;24(1):193-8. Portuguese

8. Vilela P, Duarte J, Goulão A. Doença cérebro-vascular na gravidez e no puerpério [Cerebrovascular disease in pregnancy and puerperium]. Acta Med Port. 2001;14(1):49-54. Portuguese

9. Adry RA, Lins CC, Brandão MC. Trombose venosa cerebral: relato de casos e revisão de literatura [Cerebral venous thrombosis: case report and literature review]. J Bras Neurocirurg. 2012;23(2):160-5. Portuguese

10. Kirchhoff DF, Kirchhof DC, Silva GS. Espectros clínicos da trombose venosa cerebral [The clinical spectrum of cerebral venous thrombosis]. Rev Neurocienc. 2013;21(2):258-63. Portuguese

\section{CONFLITO DE INTERESSES}

Os autores declaram não ter conflitos de interesses.

ENDEREÇO PARA CORRESPONDÊNCIA

Ana Raquel Marques

dr.ana.marques@gmail.com

Recebido em 08-05-2016

Aceite para publicação em 05-03-2017 


\section{ABSTRACT}

\section{HEADACHE IN PREGNANCY: WHEN THE FAMILY DOCTOR MAKES A DIFFERENCE}

Background: Headache is a common symptom encountered in primary health care. However, it may have features that suggest serious causes. The family doctor should be aware of this risk when faced with a patient with nonspecific symptom. The core competencies of family medicine should allow the doctor to manage uncertainty and to acknowledge various possibilities when faced with a pregnant patient with headache.

Case description: A 21 years old patient with a history of aortic dissection at age 9 years presented to a health care center at 12 weeks of gestation complaining of daily headaches. She did not have her own family physician previously. Paracetamol was prescribed for use as needed. Due to her cardiovascular history, she was referred for obstetric consultation at a local hospital. Two weeks later she returned to the health centre without an appointment complaining of severe pulsatile headache, with phonophobia, photophobia, osmophobia, and without improvement with analgesics. The patient was referred to the local hospital emergency department, where she was examined by a neurologist, who diagnosed migraine without aura. Two weeks later, she had a follow-up consultation at the hospital for the pregnancy. She complained of severe headache again and was prescribed analgesics. One week later she returned to the health centre without an appointment (at 17 weeks gestation) because of persistence of the headache. Due to the long course without improvement and multiple consultations with different doctors, contact was made with the neurologist on call at the local hospital and an emergency consultation was arranged. A magnetic resonance angiogram revealed a left lateral sinus cerebral venous thrombosis. She was admitted to the intensive care unit and was treated with enoxaparin.

Conclusion: Continuous, comprehensive care and the ability to manage health care resources gave the family physician an advantage in the management of this challenging case. This case highlights the importance of a family doctor for every patient.

Keywords: Headache; Pregnancy; Cerebral venous thrombosis. 